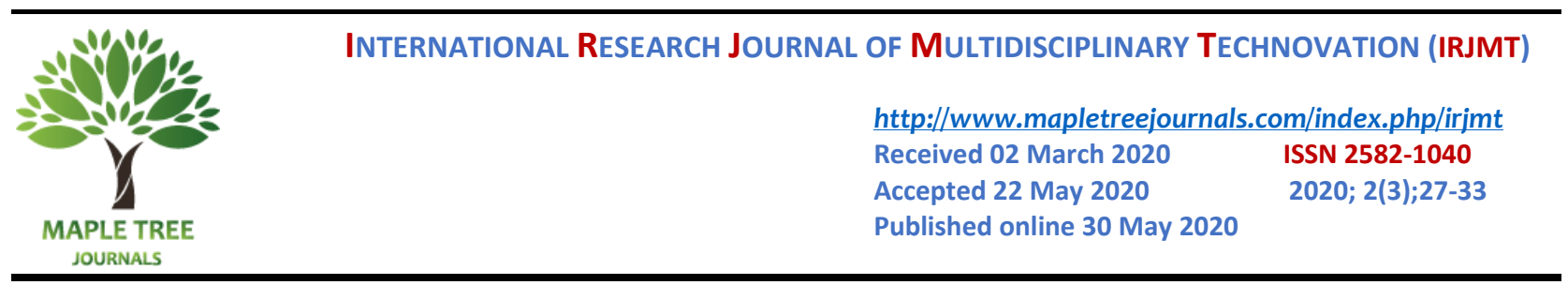

\title{
EEG Based Brain Controlled Keypad and Devices
}

\author{
R. Jeevareha ${ }^{1}$, M. Tharini ${ }^{1}$ \\ ${ }^{1}$ Department of Information Technology, Velammal College of Engineering and Technology, Madurai- 625009, \\ Tamil Nadu, India. \\ *Corresponding author E-Mail ID: jeevarehamit@yahoo.com \\ DOI: https://doi.org/10.34256/irjmt2035
}

\begin{abstract}
In our society there are many people suffer by paralytic diseases which holds them with several disabilities like unable to talk and unable to move physically and unable to express their everyday basic needs, but can still use their eyes and sometimes move their heads. This Project is under the principle of Brain Computer Interface (BCI). Our model helps them to do all their basic needs just from their wheelchair. All applications are integrated in the wheelchair, such as they can drive their wheelchair on their own using either meditation/attention or eye blink, Keypad for communication, Emergency commands for requesting their basic need such as food, water, restroom etc., Home automation for operating appliances, all those applications are done by having eye blinks using EEG device- Neurosky mindwave mobile.
\end{abstract}

Keywords: Paralyzed people, Brain Computer Interface, Wheelchair, Keypad, Emergency commands, Home automation, EEG, Neurosky mind wave mobile.

\section{Introduction}

The Brain Computer Interface (BCI) is one of the communication channels used to make an interaction between human brain and digital computer. BCI monitors EEG waves from the Brain. EEG -Electroencephalography device analyse the electrical property of the brain over the Scalp (Non-invasive). The Neuro Sky mind wave mobile measures intentionally directed EMG activity (blink strength) [1-3].

\section{Blink Talk (Keypad)}

Laptop is interfaced with the wheelchair which has the keypad used for communication by having keys. Keypad performs binary encoding of the blink patterns detected by a Neurosky mindwave mobile device and translates those codes into pre-set phrases that can be selected through speech synthesis and voiced by the host computer.

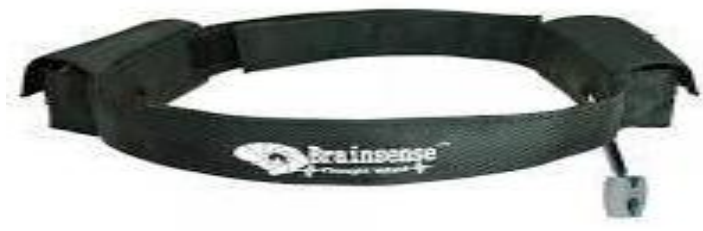

Fig 1. Neurosky Mind wave mobile device

The Neuro Sky mind wave mobile measures intentionally directed EMG activity (blink strength), which is converted into numeric values that ultimately manifest as communication with 
the user (Fig 1. Shows Neurosky Mind wave mobile device). Onscreen keyboard keys are highlighted repeatedly in a loop. When it detects a blink, the text is selected (Observes the sound occurred while blinking, to indicate that the key is selected). This transmission takes place via Bluetooth communication [5]. All the applications are designed and handled using Matlab. Fig 2. shows the keypad view.

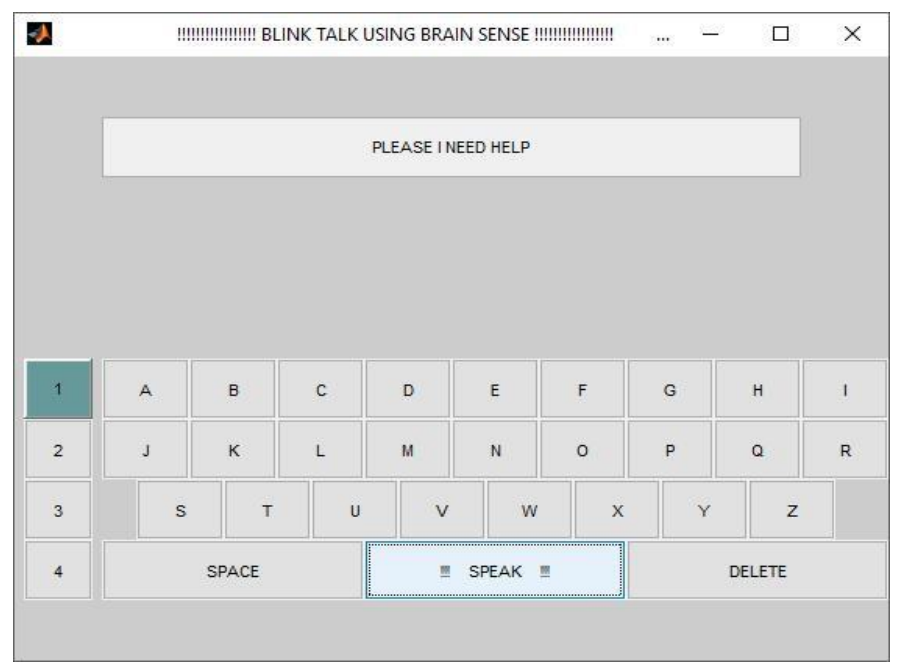

Fig 2. Blink type using keypad

\section{Block Diagram}

Since the system uses Raspberry $\mathrm{Pi}$, it does not require Matlab for processing the signal. Raspberry Pi has in-built Bluetooth, so that there is no need for external Bluetooth [6-8]. In this proposed system, virtual keyboard is designed using Python programming by having 'Tkinter' library for virtual keyboard design and 'Pyautogui' library for mouse pointer movement. Fig 3 shows Raspberry Pi Block diagram.

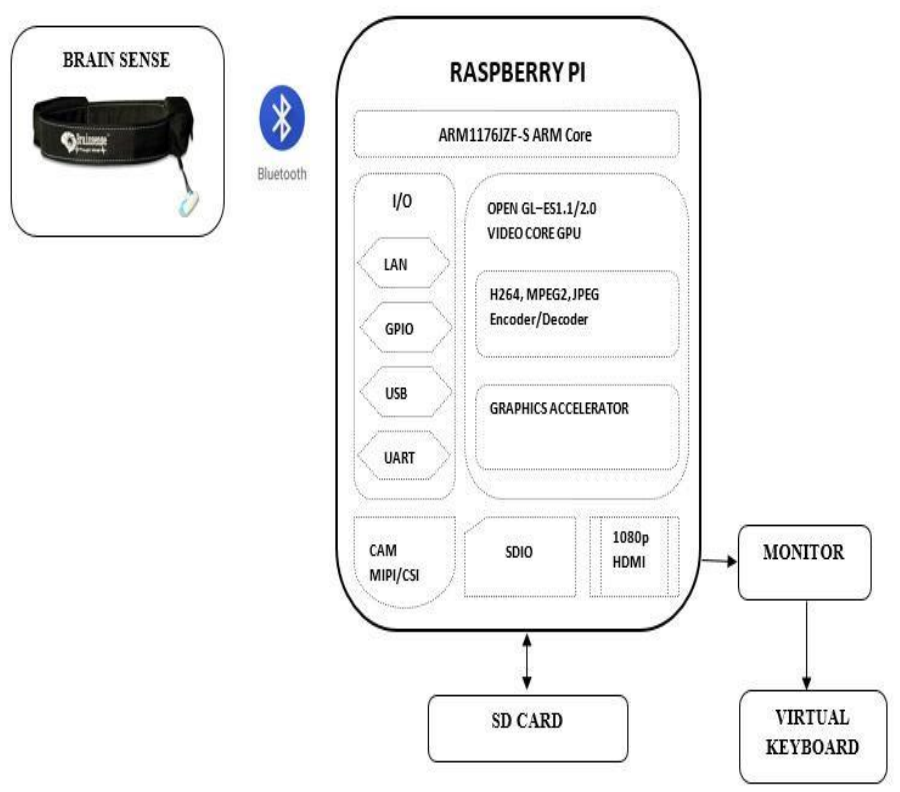

Fig 3. Block diagram 


\section{Wheelchair Control}

The people can be able to control the wheel chair to the desired place by their eye blink. So, they don't need a caretaker to drive them. They can drive their wheelchair themselves. When the wheelchair control panel opens, the direction is chosen by eye blink. The wheelchair starts moving. It also has Ultrasonic sensor which is integrated with the wheel chair to avoid collision on obstacles (example: wall). The Sensor measures the distance linearly. When it is supposed to detect any obstacle on the pathway, it starts turning left or right.

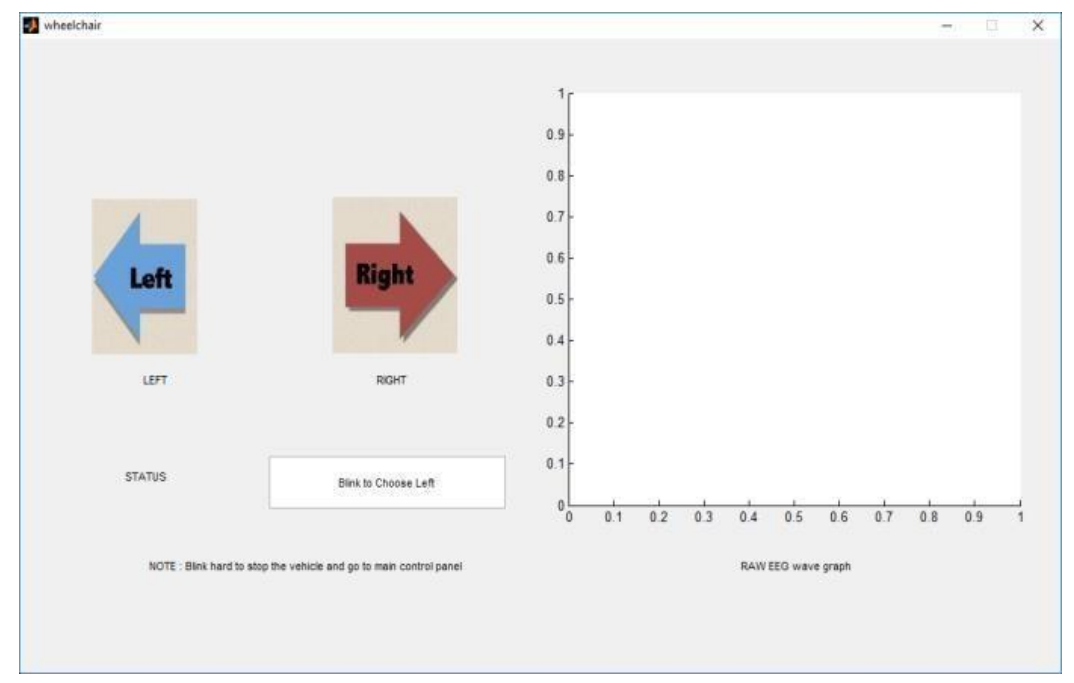

Fig 4. wheel chair control panel

\section{Ultrasonic Sensor:}

The wheel chair and the entire system is battery powered and can be recharged and used in any indoor area. At the end of the project, the following goals will be achieved.

1) To develop an EEG Based Blink detection for controlling wheel chair.

2) To develop a user interface keyboard where the user can select the keys and type using his blink so that he/she can communicate with his care taker.

3) To design a Text to Speech converter such that the keys typed or an SMS can be sent to the care taker.

4) To develop an Emergency Alert using Brain waves.

5) To design and develop an omni-directional wheel chair capable of recharging and handling a payload of $120 \mathrm{Kg}$.

6) To develop Lidar and Camera based Obstacle avoidance and Computer Vision system for smooth navigation of the wheel chair.

7) GPS based Path learning system on Neural Networks.

It should be noted that the main objective of the project is intended to evaluate the ability of the individuals to voluntarily control the wheel chair through their brain and eye activity, ensuring that the orders are executed properly [9-11].

The overall objective is to build a relatively inexpensive, easily deployable intelligent wheel chair, where user can control and communicate using this hardware.

\section{Home Automation}

Paralyzed people can be able to control the home appliances such as light, fan, TV just from the wheel chair itself using eye blinks. It is also visualized in laptop. By blinking, certain appliances can be switched ON/OFF. 


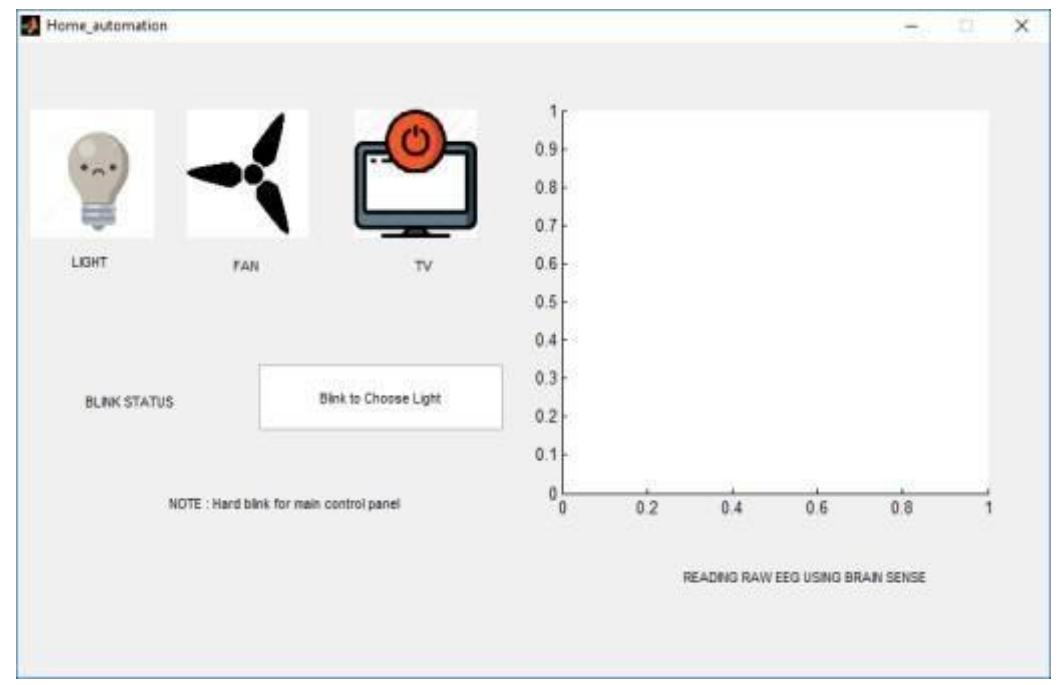

Fig 5. Home automation control panel.

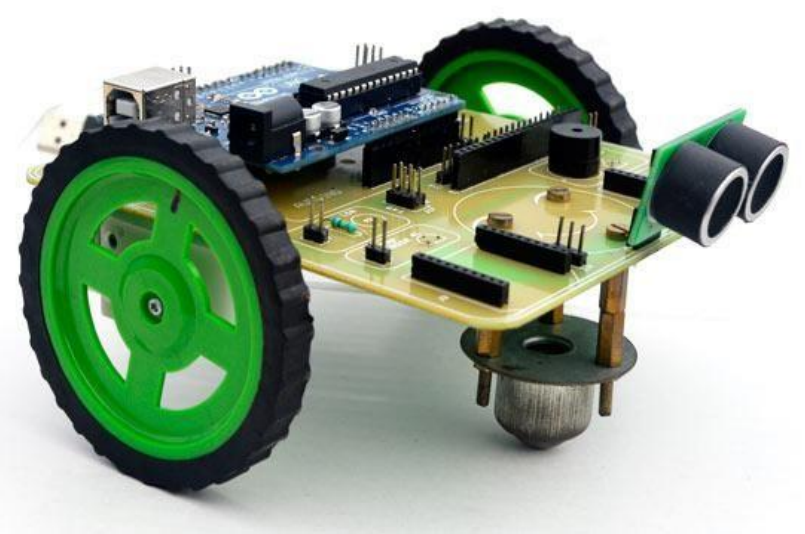

Fig 6. Wheelchair prototype

\section{Mindwave Mobile \& Raspbery Pi}

Home appliances such as light, fan, and TV which is connected with GPIO pins of Raspberry Pi through the Relay. Mind wave mobile/ Brain Sense which also has inbuilt Bluetooth, is connected with Raspberry Pi.

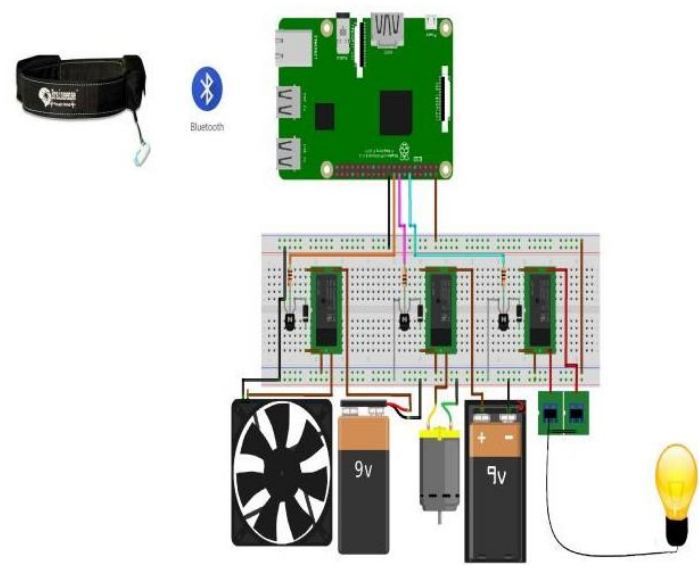

Fig 7. Automation with bulb application 


\section{Complete Wheelchair Automation}

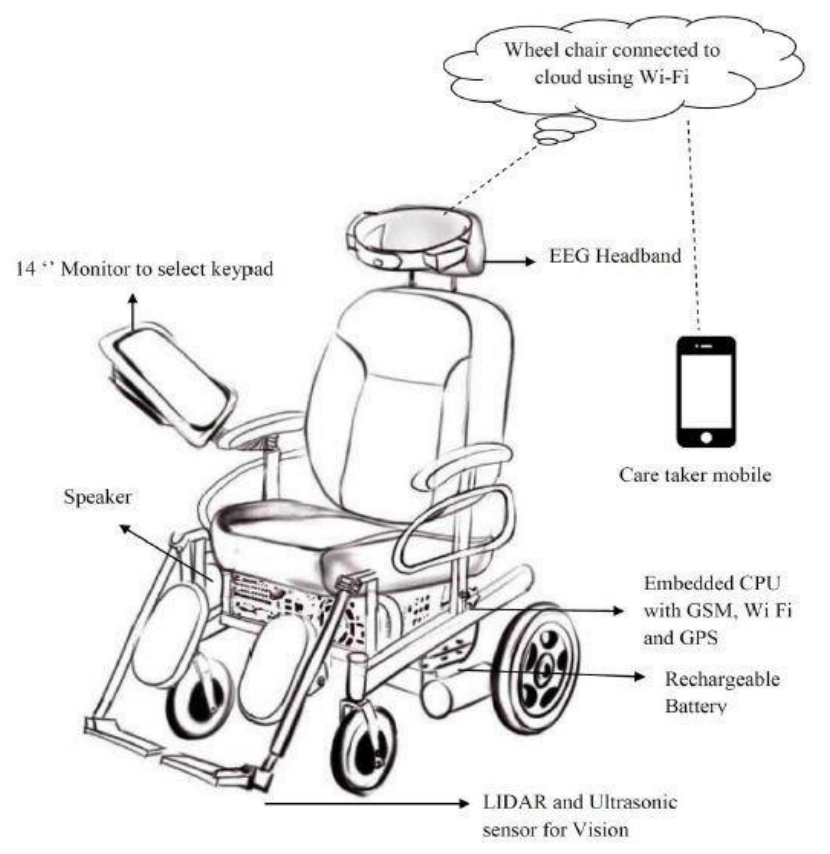

Fig 8. Fully Automated wheelchair

\section{Complete Bci Block Diagram}

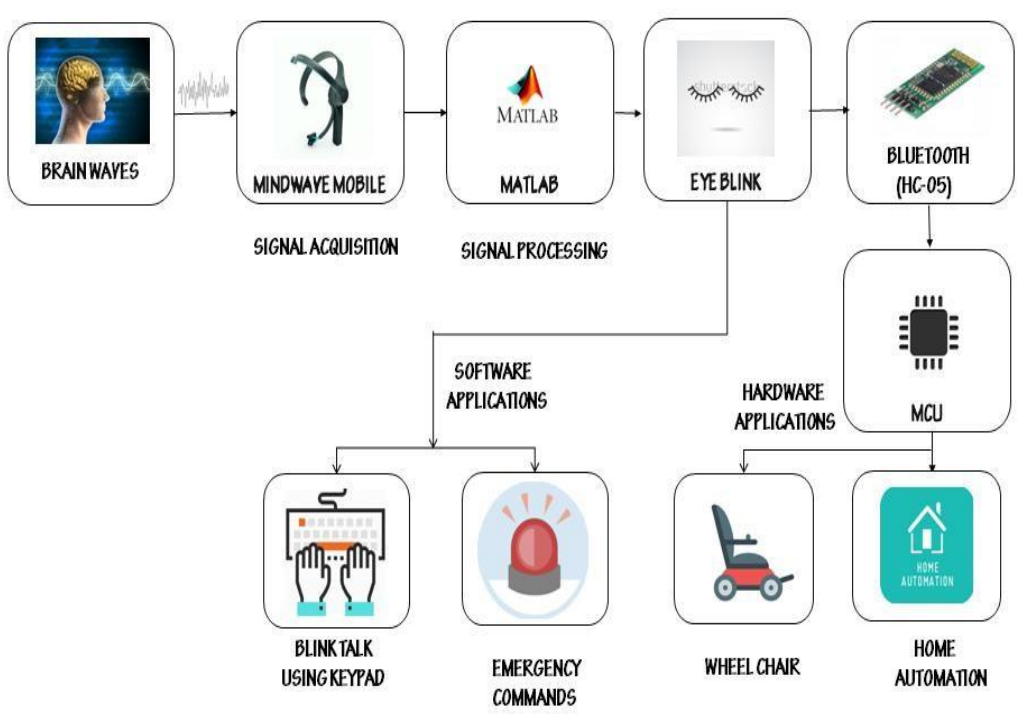

Fig 9. BCI block diagram

\section{Technology}

The technology used here is THINKGEAR technology.

Think Gear is the name of Neuro Sky's single dry sensor technology that allows the measurement, amplification, filtering, and analysis of EEG signals and brainwaves. Combined with NeuroSky's proprietary eSense algorithms, this allows a headset to be able to measure the wearer's state of mind, and makes this information available to applications so that the applications can respond to your mental activity. 


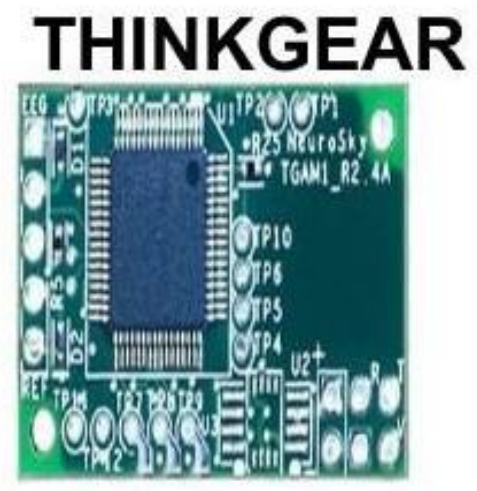

Fig 10. Thinkgear AM

The TGAM Module offers a e-sense technology. Its outputs Attention, Meditation and Physical eye blinks from raw EEG data. It communicates through UART interface at baud rate 1200, 9600 or 57600 .

\section{Existing Vs Proposed Model}

In existing model, they are using attention/ meditation for some application, it is not sure to maintain attention and meditation all the time. So, in our proposed model, we are using eye blinks for all application and mainly we merged all application with the wheel chair so that they can switch over from one application to another themselves. They no need a care taker to do all the works for them.

\section{Advantages}

[1] The NeuroSky Mindwave mobile uses a noninvasive electrode which is just placed over the scalp and so it is not necessary to pierce the scalp.

[2] It integrates all the applications in a single wheel chair such that the disable person does not need a caretaker for help.

[3] Paralyzed people can control every application using their brain from the place their place.

[4] Mainly the paralyzed people can switch over from one application to another application themselves by making 'High converged eye blink'.

\section{Conclusion}

The Brain Computer Interface uses brain signals for all applications. Since Neurosky mind wave mobile uses AAA-battery and an electrode which is just placed in the scalp. The electrode doesn't make any harm to the brain. It can be pre-configured to increase the accuracy of the application.

It can also be further developed by adding some other application such as password authentication, finding the brain state like active/ drowsy etc. and robotic arm control. It is inspired by the wheel chair used by Stephen Hawking physicist, cosmologist.

\section{References}

[1] B. Luzheng, X. A. Fan, Yili Liu, EEG Based Brain-Controlled Mobile Robots: A Survey, IEEE Transactions on Human-Machine Systems, 43 (2013) 161-176.

[2] Wei Li, Christain Jaramillo, Yunyi Li, (2012) Development of Mind Control System for humanoid Robot through a Brain Computer Interface, In $20122^{\text {nd }}$ International Conference on Intelligent Systems Design and Engineering Application, IEEE, 679-682. 
[3] J. R. Millan, F. Renkens, J. Mourino, W. Gerstner, Noninvasive Brain-Actuated Control of a Mobile Robot by Human EEG, IEEE Transactions on Biomedical Engineering, 51 (2004) 1026-1033.

[4] H.A. Shedeed, M. F. Issa, S. M. El-Sayed, (2013) Brain EEG Signal Proccessing for Controlling a Robotic Arm, In 2013 8th International Conference on Computer Engineering \& Systems (ICCES), IEEE, 152-157.

[5] S. Ramesh, M. G. Krishna, M. Nakirekanti, Brain Computer Interface System for Mind Controlled Robot Using Bluetooth, International Journal of Computer Applications, 104 (2014) 20-23.

[6] P. Deivendran, E. R. Naganathan, M. Sundaranar, Scalability Services in Cloud Computing Using Eyeos, Journal of Computer Science, 11 (2015) 254-261.

[7] A.A. Karim, T. Hinterberger, J. Richter, J. Mellinger, N. Neumann, H. Flor, N. Birbaumer, Neural Internet: Web Surfing with Brain Potentials for The Completely Paralyzed, Neurorehabilitation and Neural Repair, 20 (2006) 508-515.

[8] Hasan Bakheet Jasim, Brain Wave Technology Based Home Devices Control System, International Journal of Research, 4 (2017) 2732-2738.

[9] K.H. Solanki, H. Pujara, Brainwave Controlled Robot, International Research Journal of Engineering and Technology (IRJET), 2 (2015) 609- 612.

[10] S. Jain, A. Vaibhav, L. Goyal, (2014) Raspberry Pi Based Interactive Home Automation System Through E-Mail, In 2014 International Conference on Reliability Optimization and Information Technology (ICROIT), IEEE, 277-280.

[11] W. T. Lee, H. Nisar, A. S. Malik, K. H. Yeap, (2013) A Brain Computer Interface for Smart Home Control, In $201317^{\text {th }}$ IEEE International Symposium on Consumer Electronics (ISCE), IEEE, 35-36.

\section{Acknowledgement Nil}

\section{Funding}

This study was not funded by any grant

\section{Conflict of interest}

None of the authors have any conflicts of interest to declare.

\section{About The License}

The text of this article is licensed under a Creative Commons Attribution 4.0 International License

\section{Cite this Article}

R. Jeevareha, M. Tharini, EEG Based Brain Controlled Keypad and Devices, International Research Journal of Multidisciplinary Technovation, Vol 2, Iss 3 (2020) 27-33.

DOI: https://doi.org/10.34256/irimt2035 